fehlen neue Begriffe, um die neuen, nur teilweise urbanen Siedlungsformationen wirklich zu kennzeichnen.

Auch wenn in manchen Teilen der Welt die Städte mit der aufgrund strenger Zuwanderungsbeschränkungen und sinkender Geburtsraten zurückgehenden Bevölkerung schrumpfen, wird die überwiegende Zahl der Menschen in der Zukunft in Städten beziehungsweise urbanen Regionen leben, in denen auch der größte Bevölkerungszuwachs stattfinden wird. Die Stadt wird zum Schicksal, der Planet und die Menschheit urbanisiert. Um 1800 lebten nur wenige Prozent der Menschen in Städten, um 1900 sollen es um die 14 Prozent der Weltbevölkerung gewesen sein. 1950 gab es 86 Städte mit einer Million Einwohner oder mehr, heute sind es mehr als 400 .

Jetzt lebt bereits mehr als die Hälfte aller Menschen in Städten, in manchen Regionen wie in den Industrieländern sind dies schon 80 Prozent. Bis 2030 sind zwei Drittel aller Menschen Stadtbewohner. Noch immer wandert jeden Tag eine Million Menschen in Städte - oder in urbane Regionen. Jedes Jahr wächst so die urbane Bevölkerung hinsichtlich der Bevölkerungszahl um ein Land wie Frankreich oder in einer Größenordnung von mehreren Megacities an. Die weitere Urbanisierung findet fast ausschließlich in Ländern der Dritten Welt und in Brasilien, China und Indien statt. In diesen Ländern leben bereits etwa so viele Menschen in Städten wie insgesamt in Europa und in den USA. Und hier werden sich bis 2020 auch neun der zehn bevölkerungsreichsten Megacities befinden.

\title{
Die Stadt als Risiko
}

In einer zunehmend urbanisierten Welt wachsen mit den Städten auch die Risiken, zum Opfer von Katastrophen zu werden, gleich ob es sich um die Folgen von Terroranschlägen, Naturereignissen oder technischen Unfällen handelt. Nur mit großem Aufwand ließen sich Vorkehrungen vor manchen der Risiken treffen, die Städte betreffen können. Vor allem bei den schnell wachsenden Megacities mit über 10 Millionen Bewohnern oder urbanen Ballungsräumen in den Entwicklungsländern, in denen sich viele Menschen ungesteuert in Slums oder wilden Siedlungen niederlassen, wächst die Wahrscheinlichkeit, daß sich schwere Katastrophen ereignen können. Als 1984 durch eine Explosion in einem chemischen Werk des US-Konzerns Union Carbide 40 Tonnen hochgiftige Gase (Methylisocyanat) die indische Millionenstadt Bhopal und vor allem die 
nahe gelegenen armen Stadtviertel überzogen, starben unmittelbar zwischen 5.000 und 7.000 Menschen, in der Folge weitere 15.000 bis 20.000 Menschen an diesem Unfall, der an einen kriegerischen Akt erinnert. Er macht vorstellbar, was geschehen könnte, wenn es sich nicht um einen Unfall handelt, sondern gezielt Anschläge mit chemischen Waffen auf Städte ausgeführt würden. Auch 20 Jahre nach dem Unfall ist in Bhopal das Grundwasser noch kontaminiert, über 100.000 Menschen leiden an chronischen Erkrankungen.

Ein Jahrzehnt darauf ereilte eine andere Millionenstadt eine Katastrophe. Im Januar 1995 richtete ein Erdbeben der Stärke 7 auf der Richterskala in der japanischen Stadt Kobe gewaltige Schäden an. Über 6.000 Menschen starben, der volkswirtschaftliche Schaden war enorm. Hätte ein solches Erdbeben direkt eine Megacity wie Tokio, Mumbai, Mexiko-Stadt oder Los Angeles getroffen, dann wären die Schäden weitaus größer gewesen. Noch sind solche großen urbanen Katastrophen, verursacht durch Unfälle oder Terroranschläge, selten. Naturkatastrophen überwiegen - trotz Tschernobyl, Vesevo und anderen Industrieunfällen - bei weitem, allen voran verheerende Stürme und Hochwasser. Sie sind die häufigsten Ursachen von Schäden durch Naturereignisse, wie Wolfgang Kron von der Münchner Rück berichtet: „Rund ein Drittel aller Schadenereignisse und ein Drittel der volkswirtschaftlichen Schäden sind weltweit auf die Folgen von Hochwasser zurückzuführen; fast die Hälfte aller Menschen, die in den vergangenen Jahrzehnten bei Naturkatastrophen getötet wurden, waren Hochwasseropfer." Überschwemmungskatastrophen und die von ihnen verursachten Schäden hätten in den letzten Jahrzehnten erheblich zugenommen. Bei der Münchner Rück geht man davon aus, daß man aufgrund der Klimaveränderung, des Bevölkerungswachstums und mangelnder Vorsorge durch Bebauung gefährdeter Gebiete vermehrt mit der Zunahme solcher Katastrophen rechnen müsse, was bereits die ersten Jahre des neuen Jahrhunderts gezeigt hätten. Und in dem Anfang 2005 veröffentlichten Bericht Megastädte - Megarisiken: Trends und Herausforderungen für Versicherung und Risikomanagement macht die Münchner Rück darauf aufmerksam, daß von diesen Risiken zunehmend auch die großen Städte betroffen sein werden: „Megastädte sind allen klassischen Gefahren ausgesetzt, aber sie sind überproportional exponiert und angreifbar. Sie schaffen Risiken neuer Dimension - Megarisiken. Tsunamis zum Beispiel könnten auch Ballungsräume im Küstenbereich gefährden. Tokio und Miami etwa liegen in hoch erdbeben- beziehungsweise hurrikangefährdeten Gebieten.“ 
Gefahren treten auch dann auf, wenn es durch den Klimawandel eigentlich weniger regnet, dafür aber die Niederschläge kürzer und heftiger ausfallen und so Überschwemmungen auslösen, wie sie auch in Deutschland aufgetreten sind. Da große Städte mit weitflächig überbautem Boden und ihrer größeren Abgabe von Hitze sich sozusagen eine eigene Klimaglocke schaffen, können dadurch auch Unwetterschäden verstärkt werden, wie die Versicherungsgesellschaft erklärt: „Besonders über dicht bebauten Stadtgebieten - also Gebieten mit hoher Wertedichte - können sich durch die verstärkte Konvektion lokale Unwetter manchmal geradezu explosionsartig entladen und extreme Niederschlagsintensitäten auslösen. Diese sind oft verbunden mit hohen Blitzdichten, Hagelschlag und orkanartigen Böen, manchmal bis hin zu Tornados. Wegen des hohen Versiegelungsgrads in urbanen Gebieten strömt der Starkregen direkt zu den städtischen Entwässerungssystemen, die dafür nicht ausgelegt sind, so daß Unterführungen, Keller und manchmal auch U-Bahn-Schächte mit Wasser vollaufen." Tokio (35 Millionen Einwohner) erstreckt sich beispielsweise über eine Fläche von 13.000 Quadratkilometern, Los Angeles gar über 14.000 Quadratkilometer, obgleich hier mit 16 Millionen Einwohnern nicht einmal halb so viele Menschen leben. Begreift man das Ruhrgebiet (11 Millionen Bewohner) als urbanen Ballungsraum, so überzieht dieser auch fast 10.000 Quadratkilometer und ist damit größer als die Fläche, die Megacities wie Mumbai (20 Millionen), São Paulo (20 Millionen) oder Mexiko-Stadt (22 Millionen) bedecken, deren Stadtgebiet nur zwischen 4.000 und 5.000 Quadratkilometer umfaßt.

In schneller Folge sind 2005 Städte zu Opfern von Naturkatastrophen geworden. Eingeleitet wurde die Serie vom Tsunami in Südostasien, der mit unterschiedlicher Kraft der Verwüstung die Küstenregionen in Sri Lanka, Indien, Bangladesch, Burma, Thailand und Indonesien betroffen hat und auch geographisch bereits eine internationale Katastrophe war. In dem von von Cordt Schnibben herausgegebenen Buch Tsunami - Geschichte eines Weltbebens (München, 2005) wurde gar von einem „Weltbeben“ gesprochen, vielfach galt sie als „Jahrhundert-Katastrophe“, wobei diese quantitative Einstufung auf einer durchaus ambivalent zu verstehenden Hit-Liste schon auf die Erwartung einer weiteren Steigerung hinweist. Trotz der hohen Opferzahlen von vermutlich über 200.000 Toten sowie von Millionen Obdachlosen blieben die großen Städte weitgehend verschont. In der Erinnerung aber dürften bei vielen die Bilder der Städte besonders auf Sumatra wie Banda Aceh geblieben sein. Die Stadt mit mehr als 300.000 Einwohnern war von der Flutwelle auf einen 
Schlag zu großen Teilen einfach von der Landkarte gelöscht worden. Symbolisch sah man auf einer freigeräumten Ebene, auf der zuvor noch ein Stadtviertel war, nur noch den Turm einer Moschee, der den Fluten widerstanden hatte. Ansonsten war die weitgehend zerstörte Stadt voller Trümmer, Unrat und Leichen, dazwischen irrten Überlebende herum. Apokalyptische Bilder für die Menschen in den Wohlstandsfestungen wohl auch deswegen, weil sie nicht nur mehr oder weniger live die Bilder des Schreckens über das Fernsehen und das Internet ins Haus geliefert bekamen, sondern auch, weil viele die Region als „Urlaubsparadies“ kannten, über dessen Idylle nun die Katastrophe hereingebrochen war. Auch wenn zuvor schon Entführungen und Anschläge von Terroristen Touristen und ihren Urlaubsorten in Bali, Tunesien, Kenia oder Ägypten galten, war dieser „Terrorangriff der Natur" auf die Zufluchtsstätten der Menschen aus den reichen Ländern doch ein Einschnitt und auch eine Erinnerung daran, daß Naturkatastrophen, darin Terroranschlägen ähnelnd, sich immer und überall plötzlich ereignen können, daß es keine sicheren Orte und Regionen auf der Welt gibt - und natürlich, daß die Orte, die am dichtesten bevölkert sind, von Katastrophen am schlimmsten betroffen sein können, während sie zugleich die begehrtesten Terrorziele sind.

Der Tsunami in Südostasien brachte ein Ereignis wieder in Erinnerung, das wie wenige andere sonst die Geschichte der Moderne skandiert und geprägt hat. Wie in Südostasien folgte auch dem Erdbeben von Lissabon, der damals viertgrößten europäischen Metropole, am 1. November 1755 eine gewaltige Flutwelle, die einen Großteil der Stadt und der übrigen Siedlungen an der Küste zerstörte. 60.000 Menschen, manche sagen auch 100.000, sind vor allem durch die Flutwelle getötet worden, die eine Höhe zwischen 6 und 20 Meter erreicht haben soll. Sie hat viele tausend Menschen in den Tod gerissen, die zunächst vor dem Erdbeben aus der Stadt an den Tejo geflüchtet waren. Was Erdbeben und Flutwelle nicht zerstörten, sollte das Feuer vernichten, das eine Woche lang in der Stadt wütete. Lissabon war eine große Stadt in einem kleinen Land am Rande Europas, dank der Ausbeutung Lateinamerikas und des Welthandels eine blühende und reiche Handelsmetropole, die plötzlich nicht mehr existierte.

Man lernte damals schnell, schaffte offenbar ein gutes Katastrophenmanagement und begann damit, den Schutt zu beseitigen und das neue Lissabon aufzubauen, während die Katastrophe den Grundstein für die Seismologie legte, für die Wissenschaft der geologischen Katastrophen. 
Es dauerte lange, bis sich die Nachricht über die zerstörte Stadt herumsprach; heute ist das nur noch eine Frage von Minuten. Von den 250.000 Einwohnern kostete das Erdbeben von Lissabon zwischen 50.000 und 100.000 Menschen das Leben. Schäden durch zusammenstürzende Gebäude sowie durch die Flutwelle gab es auch an der Algarve und in Spanien. Bemerkt wurde das Beben fast in ganz Europa, die Flutwelle konnte angeblich sogar noch in Südamerika oder in Finnland wahrgenommen werden. Den einen galt die Katastrophe als ein göttliches Strafgericht, das wieder einmal ein Babylon ereilt hat; für die Rationalisten unter den Aufklärern war es vor allem eine geistige Erschütterung. In die beste aller möglichen Welten, der Theodizee der Aufklärung, die mit dem Glauben an die ehernen Gesetze der Natur und den kontinuierlichen Fortschritt des Geistes verbunden war, mußten nun Katastrophen eingebaut werden. Das schmälerte einerseits die der Vernunft zugeschriebene, auf fest gefügten natürlichen Ordnungen ruhende Kraft, eröffnete aber andererseits mit der Abwendung von der Gewißheit und dem Immerwährenden zur Berechnung des Wahrscheinlichen auch den Vorstellungshorizont von einer kreativen Destruktion, einer Ästhetik des Erhabenen und eines sozialen Bebens, einer gesellschaftlichen Revolution, die schließlich auch zum Umsturz der gesellschaftlichen Ordnung führte.

Die Natur hatte sich zurückgemeldet, bedrohte die Fundamente der Vernunftarchitektur und betraf nicht mehr nur die Menschen auf dem Land, sondern eben auch die in den naturfernen, künstlichen urbanen Welten, die trotz aller, von den modernen Medien nur verstärkten mentalen Attraktionen durch reale und vorgestellte Katastrophen im immer kurzen Gedächtnis vor den Naturgewalten sicher zu sein schienen. Die Angstlust am Untergang hatte sich allerdings schon seit dem Aufkommen von Städten mit diesen verbunden, weil deren Zerstörung real und in der Vorstellung durch Naturkatastrophen, von Gott oder Menschenhand verursacht beeindruckende Spektakel bot: Atlantis, Babylon, Sodom und Gomorrha, Troja sind nur einige der mythischen Vorbilder für die zugleich imposanten und riskanten räumlich verdichteten Stadtgebilde, die das Land beherrschten und sich mit mächtigen Mauern schützten, hinter denen sich eine neue Kultur mit einer außerordentlichen Dynamik und einer künstlichen Wirklichkeit herausbildeten.

Man konnte nicht mehr davon ausgehen, daß der Fortschritt einfach immer so weiter geht, daß die Natur ein von Technik und Wissenschaft irgendwann endgültig gezähmtes Objekt sein werde. Seitdem ist das Wissen um die Unvermeidbarkeit von Naturkatastrophen größer geworden. 
Aber es hat sich, zumindest in den reichen Ländern, mit den permanenten Warnungen vor neuen Epidemien, gigantischen Flutwellen, Vulkanausbrüchen, Erdbeben, die Megacities bedrohen, möglichen Einschlägen von Asteroiden und all den Folgen, die mit dem Umbau der Ökosysteme, der wachsenden Weltbevölkerung und einer Klimaerwärmung einhergehen können, wieder ein trügerisches Gefühl von Sicherheit eingestellt, das mit Beginn des 21. Jahrhunderts nun Schlag auf Schlag zu erodieren scheint. Eingeleitet von den Anschlägen vom 11. September und den Szenarien der Bedrohung durch Massenvernichtungswaffen, die - wie im Kalten Krieg die Atombombe - vornehmlich auf die räumlich verdichteten Städte gerichtet sind, über den Tsunami von Südostasien, drohende Grippeepidemien, das Erdbeben, das weite Teile von Kaschmir betraf, auch zahlreiche Städte, aber keine Megacities, bis hin zu den Orkanen, die 2005 in gehäufter Folge über die Karibik, Mittelamerika und die USA zogen und in den USA auch wieder die Verletzlichkeit moderner Gesellschaften vor Augen führte.

Die Schäden, die der Hurrikan Katrina in New Orleans verursacht hat, waren vorhersehbar. Nicht vorhergesehen wurden jedoch die Folgen, die in einer Gesellschaft entstehen, die nicht nur tief sozial und ethnisch geteilt ist, sondern in der sich der Staat auch aufgrund der damit verbundenen Ideologie der Eigenverantwortung weit aus der systematischen Vorsorge für seine Bürger zurückgezogen hat und eher schlecht als recht Nothilfe organisiert - vergleichbar den Spenden von Privatpersonen oder Unternehmen, die zwar Hilfe leisten, aber strukturell nichts verändern und mit deren Geldern nicht gerechnet werden kann. Die Flutwelle zog so plötzlich die Decke über einer Stadt weg und gab den Medien und damit der Öffentlichkeit den Blick auf die meist vergessenen Stadtviertel und ihre verarmte Bevölkerung frei. Wer konnte, floh. Wer nicht über finanziellen Mittel und kein Auto verfügte, blieb zurück. Aus dem Sturm und der Flut tauchten schließlich die Menschen auf, die in der Wirtschaft und Gesellschaft keinen Platz finden, und dazu die Orte, die normalerweise weder die wohlhabenderen Bewohner noch gar Touristen betreten. Viele der Bewohner kamen aus diesen armen Vierteln oder Ghettos mit den schlechten Adressen und den verbauten Aussichten kaum jemals heraus.

Wie in New Orleans fallen in den urbanen Druckkesseln schnell die Hemmschwellen, bricht Gewalt und Zerstörungswut aus und kommt es zu Plünderungen. New Orleans aber ist eine relativ kleine Stadt in einem reichen Land. Trotz vieler Probleme und einer viel zu spät einsetzen- 
den staatlichen Katastrophenhilfe wurden schließlich Hunderttausende Obdachlose, die zeitweise oder auf Dauer nicht mehr in ihre Häuser und Wohnungen zurückkonnten, woanders untergebracht. Dazu kamen die Menschen, die ihre Arbeit oder ihre Geschäfte verloren haben. Bei einer Megacity hingegen hätten Millionen auf einen Schlag fortgebracht und versorgt werden müssen. Kaum vorstellbar, wie dies je geschehen soll. Aber die Möglichkeit ist durchaus konkret. Schon jetzt leben 100 Millionen Menschen beispielsweise in Gebieten, die unter dem Meeresspiegel liegen oder überflutet werden können. Darunter eben auch Megacities, in die immer mehr Menschen strömen und deren Slums besonders bedroht sind. Steigt aufgrund der Klimaerwärmung, wie befürchtet, der Meeresspiegel an und nehmen die Stürme an Zahl und Heftigkeit zu, muß man mit einer großen Katastrophe rechnen. Werden dann zig Millionen zu „Umweltflüchtlingen“, wie eine neue, von der UNO geprägte Kategorie heißt, so dürften, abhängig vom Ort der Flutkatastrophe, die Probleme der großen Städte mit einem abrupt anschwellenden Strom von Migranten noch einmal drastisch anwachsen. Auch die langsam voranschreitende Umweltzerstörung wird dazu führen, daß die Migrantenströme vor allem in die Städte noch einmal anwachsen. Die UNO befürchtet, daß in den nächsten Jahrzehnten durch die Klimaerwärmung, aber auch durch Raubbau, Bevölkerungszunahme und als Folge der Globalisierung bis zu 30 Prozent der Landflächen zu Wüsten werden können. Das drängt die Menschen noch weiter als bisher zusammen und intensiviert den Druck auf das Land und das Wachstum der Städte.

Nach Angaben der UNO hat sich beispielsweise die Bevölkerung von Sana'a, der Hauptstadt Jemens, seit 1972 alle sechs Jahre verdoppelt. Nun liegt sie bei etwa 900.000 , aber das Grundwasser sinkt jährlich um sechs Meter. 2010 wird es erschöpft sein. Aber auch andere Veränderungen vollziehen sich mit großer Geschwindigkeit. Die Wüste Gobi in China vergrößert sich jedes Jahr um 10.000 Quadratkilometer. Die von ihr ausgehenden Sandstürme reichen bis Korea oder Japan. Die Wüste schiebt sich auch in den nordafrikanischen Ländern wie Marokko oder Algerien immer weiter vor. In Ägypten ist die Hälfte des bewässerten Landes bereits versalzen. Auch in den USA gibt es dramatische Veränderungen. So verliert Louisiana jährlich 65 Quadratkilometer Land an das Meer. In Europa wird im Mittelmeerraum der Wassermangel in den nächsten Jahrzehnten steigen. Durch Trockenheit, Waldbrände, Erosion sowie eine falsch betriebene Land- und Forstwirtschaft und intensiver Bewässerung droht mindestens ein Drittel Spaniens zur Wüste zu wer- 
den. Schon jetzt leidet die iberische Halbinsel unter einer extremen Trokkenheit und Hitze. Wenn es weniger schneit, werden auch im Mittelmeerraum die Flüsse im Sommer weniger Wasser führen und wird das Grundwasser absinken.

New Orleans als Opfer von Katrina ist nicht vergleichbar mit anderen Städten, aber es ist dennoch ein weiteres Beispiel dafür, mit welchen Risiken die urbanisierte Gesellschaft in Zukunft mit steigendem Meeresspiegel und häufigeren Stürmen zu rechnen hat. In Zukunft muß damit gerechnet werden, daß Katastrophen wie beim Tsunami Ende 2004 weitaus größer ausfallen werden, wenn viele Millionen Menschen gleichzeitig zu Opfern werden, fliehen oder evakuiert werden müssen. Eine Massenkatastrophe, die eine Metropole und damit Millionen Menschen auf engstem Raum betrifft, wäre kaum zu bewältigen. Bei dem „Test“ durch Katrina, vier Jahre nach dem 11. September, wurde die Frage gestellt, ob die amerikanische Gesellschaft auf eine große Katastrophe vorbereitet ist. Die Regierung im reichsten Land der Erde hat versagt. Die vom Orkan verursachten Schäden waren vermutlich schwerer, als dies bei kurzfristig vorhersehbaren Terroranschlägen der Fall wäre, selbst wenn schmutzige Bomben oder chemische Waffen verwendet würden. Aber man hatte sich tagelang vorbereiten können, was bei einem Terroranschlag nicht möglich ist.

Nach dem Hurrikan standen in New Orleans große Teile unter Wasser. Die Bilder aus der überschwemmten Stadt erweckten bei Kommentatoren nicht nur Eindrücke, wie sie bei Katastrophen in Dritte-Welt-Ländern entstehen, sondern auch solche, die an apokalyptische Filmbilder erinnerten. Menschen saßen auf Dächern, Leichen trieben im Wasser, Plünderer holten, was sie finden konnten, Gewalt brach aus, die Vertreter der staatlichen Macht und Ordnung waren hilflos oder zeigten sich in manchen Vierteln zunächst gar nicht. Die Reichen und die Menschen aus der Mittelklasse waren aus der Stadt geflohen, die nicht überfluteten Teile der Stadt, in denen die Wohlhabenden leben, wurden aus der Luft und auf dem Land versorgt, Besitz und das Leben durch private Sicherheitskräfte geschützt. Schwer bewaffnete Polizisten und Soldaten beherrschten tagelang das Stadtbild. Die untergetauchte Stadt war zu einem gefährlichen Terrain geworden. Auch wenn nach Tagen des Chaos schließlich Polizei und Nationalgarde für Ordnung sorgten, war die Stadt dort, wo sie überflutet war, zu einer mitunter gefährlichen Müllkippe geworden. Das ist die andere Seite auch von reichen Städten und Stadtvierteln, in deren Infrastruktur, Gebäuden und Fabriken, Materialien und Waren potenti- 
elle Schadstoffe lauern. In einer Stadt wird nicht mehr Gebrauchtes oder Überschüssiges normalerweise kontinuierlich hinausgeschafft. Der Müll landet in Deponien, wird verbrannt oder bestenfalls recycelt. Im Falle einer Katastrophe zeigt sich die technische Zivilisation hingegen als Produzent von riesigen Mengen riskanten Mülls. In New Orleans handelte es sich um Millionen von Kubikmetern von Abfall, der langwierige Probleme der Entsorgung schafft, sollten die vermüllten Stadtteile je wieder benutzt werden.

Das Wasser war zunächst wie nach einem Angriff mit biologischen Waffen mit Krankheitskeimen und wie nach einem Anschlag mit chemischen Waffen mit Öl, Schwermetallen, Herbiziden und anderen gefährlichen Rückständen verseucht. Das wäre auch in jeder anderen Stadt so. Einige starben an Krankheiten, die durch das verseuchte Wasser verursacht wurden. Schiffe, Autos, Tanks, Rasenmäher und andere Maschinen hatten gewaltige Mengen an Öl ins Wasser abgegeben. Dazu kam Öl aus überfluteten Raffinerien und beschädigten Ölbohrtürmen. Aus den überfluteten oder zerstörten Häusern dürfte sich eine Flut von Reinigungsmitteln, Farben, Asbest, Batterien und elektronischen Geräten stammenden Schadstoffen über das Land und im Wasser verbreitet haben. Dazu kamen gefährliche Substanzen aus Mülldeponien und von Industrieanlagen. Die DeLisle-Fabrik von DuPont, dem zweitgrößten Hersteller von Titanium-Dioxid in den USA, wurde überflutet. Die Fabrik hinterläßt jährlich 7.000 Tonnen Giftabfall, der teilweise in Müllplätzen gelagert wird, die mit der Flut ihre Inhalte preisgaben. Lois Gibbs, Direktor des Center for Health, Environment and Justice, fragte einige Tage nach der Flut: „Die ganze Stadt ist jetzt eine gefährliche Müllhalde. Wie säubern wir eine ganze Stadt, eine ganze Region?“

Anfang November 2005 waren wieder zwischen 60.000 und 100.000 Menschen in die Stadt zurückgekehrt, aber es handelte sich weitgehend um Weiße und Menschen aus dem Mittelstand. Damit hatte sich zumindest vorerst die Bevölkerungsstruktur radikal verändert, denn vor der Flut waren zwei Drittel der Bewohner schwarz. Das French Quarter und die höher gelegenen reicheren Viertel waren schnell wieder zugänglich, aber der Großteil der Stadt war zwei Monate nach der Katastrophe noch vermüllt und ohne Strom. 80 Prozent der geflüchteten und evakuierten Menschen, in der Mehrzahl Schwarze, lebten noch Monate darauf in Hotels, in neuen Wohnungen oder in Wohnwagen. Viele werden nicht mehr zurückgehen können, viele wollen auch gar nicht mehr. $\mathrm{Ob} \mathrm{New}$ Orleans wieder einmal eine größere Stadt werden oder vielleicht nur 
ein kleinerer Touristenort mit weitgehend weißer Bevölkerung bleiben wird, ließ sich selbst im Januar 2006 noch nicht sagen. Allerdings war das ehemalige New Orleans wegen der hohen Armut auch eine gefährliche Stadt gewesen. Jährlich gab es hier 72 Morde auf 100.000 Einwohner, in New York sind es zum Vergleich sieben. In New Orleans soll es, wie es in Zeitungsberichten hieß, Tausende von Kriminellen, Drogenhändler und Gangs gegeben haben. Sie seien mit den Einwohnern geflüchtet und bislang nicht wiedergekommen. Nach der Flut und mit dem Auszug der Armen und Überflüssigen sei, so der Polizeichef Warren J. Riley Ende Dezember 2005, zu einer der sichersten Städte in den USA geworden. New Orleans wurde durch den Hurrikan zu einer überschaubaren Kleinstadt.

New Orleans demonstrierte, daß der Terrorismus gegenüber dem, was Naturkatastrophen anrichten können, ein vergleichsweise kleines Problem ist. Die Bilder von der zeitweise untergegangenen Stadt wiesen aber auch auf die Hilflosigkeit eines Staates hin, der ungeheure Ressourcen in die Abwehr der von Terroristen ausgehenden Gefahr für das Land steckte und dann plötzlich hilflos und wie gelähmt dasteht, wenn die Bedrohung aus einer unvermuteten Richtung kommt.

Wenn sich nicht gerade eine Katastrophe ereignet hat, werden in einer vom medialen Kurzzeitgedächtnis beherrschten Gesellschaft Ressourcen von den die Medien und die kollektive Aufmerksamkeit weniger ansprechenden Aufgaben abgezogen. Verbesserungen der Infrastruktur, Schutz vor Naturkatastrophen oder Sicherung der gesellschaftlichen Solidarität oder des sozialen Friedens durch den Umbau der Städte und Sozialprogramme liefern keine aufregenden Bilder. Und selbst wenn eine Naturkatastrophe geschieht, wird sie zwar kurz „prominent“ und wirkt verstörend, aber verliert schnell - abgesehen von den unmittelbar Betroffenen - an Aufmerksamkeit, da es ein Geschehen ohne Täter ist, auch wenn es im Hinblick auf die Gefahrenabwehr Verantwortliche für das Nichtstun gibt. Aber Nachlässigkeit ist eben etwas anderes als mörderische Absicht.

Die absehbare Naturkatastrophe in Form des Hurrikans Katrina hat in den USA, deren System ganz auf Terrorabwehr und -bekämpfung ausgerichtet war, die Dimensionen wieder zurechtgerückt. Deutlich wurde, daß - bislang wenigstens - der real existierende Terrorismus gegenüber den Gefahren verblaßt, die von Naturkatastrophen ausgehen. Hier aber sind militärische Strategien ebenso wenig gefragt wie PsyOp-Inszenierungen, das populistische Drama von Guten und Bösen verfängt ebenso 
wenig wie die Drohung, daß derjenige, der nicht für uns ist, automatisch zum Feind wird. Just im Medienzeitalter muß sich die urbanisierte, also hoch räumlich verdichtet lebende Weltbevölkerung darauf einrichten, mit einem „Feind“ umzugehen, der teilweise wie im Fall des Terrorismus das Produkt der eigenen Handlungen ist, aber auch unabhängig von den Menschen zuschlägt. Mit einem „Feind“, der nicht „auszurotten“ ist, dessen Folgen aber reduzierbar wären, wenn die Menschen vorsorgen und ihre Lebensweise verändern. Nicht nur die Waffen sind gefährlich, deren Einsatz denkbar wäre, die Lebensweise selbst, allen voran die Urbanität, zeigt sich als eine riskante Daseinsform, als ein Aufbruch der Menschen in eine von der Natur gelöste Daseinsform und zugleich als ein Abenteuer, als ein Tanz auf dem Vulkan. Viele der Metropolen und Megacities, die an riskanten Orten und vor allem in Meeresnähe sich befinden, werden dort bleiben, denn es ist auch nicht in den kühnsten Vorstellungen denkbar, daß sich Umsiedlungen von vielen Millionen Menschen planen und ausführen ließen. Das wäre selbst in reichen Ländern weder finanziell noch logistisch zu bewältigen.

Auch ein aus einem mutierten Vogelgrippevirus H5N1 entstehender, für die Menschen gefährlicher Erreger wäre wie alle ansteckenden Seuchen eine besondere Bedrohung für Städte. Hier leben nicht nur Millionen, hier gibt es eben auch die oft besonders dicht bewohnten Orte, Ghettos und Slums, deren Bewohner von staatlichen Behörden oft nicht gut erreichbar sind oder die den Kontakt mit diesen scheuen. Eine leicht übertragbare Infektion wie die Grippe - oder in der Neuzeit die Pest - breitet sich in Städten weitaus schneller als auf dem Land aus. Und weil die Städte auch Verkehrsknotenpunkte sind, können sich Seuchen national und global schnell auf weitere Städte und damit weltweit verbreiten. Man kann zwar einzelne Menschen unter Quarantäne stellen, vielleicht auch Dörfer und kleine Städte, aber nicht Großstädte und Megacities.

Für Krankheitserreger ist die durch die Waren- und Menschenströme verbundene Welt zu einem einzigen globalen Lebensraum, zu einer globalen Stadt, geworden. Hunderte von Millionen Touristen jährlich verbinden die Länder und Kontinente auch im Sinne der Viren und Bakterien, die mit Menschen, Tieren, Waren oder Futter mitreisen und neue Nischen finden. Mit der Zerstörung von Lebensräumen von Tieren ist nach Ansicht von Experten überdies mit einer Vermehrung von Infektionskrankheiten zu rechnen, bei denen die Erreger wie beim Ebola-Virus den Wirt wechseln und sich Menschen anpassen können. Von den mehr als 1.400 bekannten menschlichen Pathogenen stammen 61 Prozent von 
Tieren. Wissenschaftler gehen davon aus, daß bislang nur ein Fünftel oder gar nur ein Fünfzigstel der Pathogene, die für den Menschen gefährlich werden könnten, bekannt ist. Ein Überspringen der Pathogene von wild lebenden Tieren ist zwar vermutlich im Laufe der Geschichte oft geschehen, aber meist seien die infizierten Menschen gestorben oder hätte sich die Epidemie in dünn besiedelten Gebieten nicht weiter verbreiten können. Das aber sei heute grundlegend anders. Die Zahl der Menschen nimmt weiter zu, sie breiten sich auch in die bislang noch wenig besiedelten Gebiete aus, und der Reise- und Güterverkehr macht aus einst lokalen Epidemien wie beim West-Nil-Virus globale.

Die chinesische Regierung hatte 2003 zunächst das tatsächliche Ausmaß von Sars gegenüber der Weltöffentlichkeit verschwiegen, nicht schnell genug an der Lungenentzündung Erkrankte unter Quarantäne gestellt und kein Reiseverbot über die Gebiete verhängt, die davon betroffen waren. Hinausgezögert wurden damit auch die Identifizierung des Erregers und die Entwicklung eines Gegenmittels. Schnell wurde die Krankheit dann nach Europa und Kanada verschleppt. Die Angst vor Anstekkung, besonders in den USA, wo Erinnerungen an die Anthrax-Briefe wieder wach wurden, führte zu Reaktionen wie der Forderung „Isoliert China!“ (Titel eines Artikels im Wall Street Journal). Wenn Sars eine so hohe Ansteckungsgefahr gehabt hätte und so gefährlich gewesen wäre wie ein neuer Grippevirus, wären die ergriffenen Maßnahmen wohl zu spät gekommen. So verlief die Epidemie, auch dank der weltweiten Kooperation, relativ harmlos. Wie nach dem 11 . September fast schon üblich geworden, wurde von manchen zuerst ein Anschlag mit einer neuen Biowaffe vermutet. Aber auch so ging in Chinas Städten die Panik um. Die Menschen bewegten sich, wenn sie denn wieder an die Öffentlichkeit gingen, nur mit Atemmasken. Taxis, Busse, U-Bahnen und Flugzeuge wurden ebenso wie öffentliche Plätze, Bahnhöfe, Büros und manchmal auch Wohnhäuser desinfiziert. Die Straßen, Plätze, Märkte und Kaufhäuser in Peking waren tagelang leer. Schulen und Universitäten waren geschlossen, die 13 Millionen-Stadt befand sich in einem Ausnahmezustand. In Krankenhäusern, in denen Infizierte in Quarantäne gehalten wurden, durfte auch das Personal die Gebäude nicht mehr verlassen. Kliniken in Peking wurden beispielsweise mit „Videophonen“ ausgestattet, damit die Angestellten mit ihren Angehörigen ohne körperlichen Kontakt in Verbindung bleiben konnten. Bewohner mehrerer Dörfer in der Nähe Pekings errichteten im Mai 2003 Straßensperren aus Steinen und Erde, um niemanden mehr durchzulassen. 
Mehr denn je sind Städte und urbane Regionen das Schicksal der Menschen, die trotz Geburtenrückgang in den reichen Ländern die Erde mit ihrer milliardenfachen Präsenz verändern und durch ihre Konzentration in Megacities auch die Dimensionen der Katastrophen entsprechend vergrößern. Dem globalen Trend der Urbanisierung läuft allerdings die Technik entgegen. Anders als vor 10.000 Jahren ist die räumliche Verdichtung in Städten keine gesellschaftliche Notwendigkeit mehr. Dadurch wird das Risiko, das vom Leben in Städten ausgeht, aber nur noch deutlicher.

\section{Eine Kurzgeschichte der Stadt im Krieg}

Für einen Großteil der Geschichte der Urbanität boten Städte allerdings Schutz vor Angriffen und galten als Orte, gegen die man keinen Krieg führen sollte, weil dies zu hohe Verluste mit sich brachte. Die Stadt, das ist eben auch für lange Zeit der durch und durch künstliche Lebensraum einer vor dem Außen durch Mauern geschützten und abgegrenzten Insel im Land gewesen. Wenn die Mauern ausreichend dick und hoch waren und für Ernährung der Einwohner vorgesorgt worden war, sich kein Verrat ereignete oder die Einwohner anderweitig überlistet werden konnten, boten Städte über Jahrtausende auch gegen zahlenmäßig weit überlegene Gegner einen hohen Schutz.

Bis zur Erfindung des Schießpulvers und damit mächtiger Distanzwaffen mußten Angriffe auf die Stadt seitens der Belagerer stets aus relativer Nähe geführt werden. Geschosse konnten schon wegen ihres Aufprallwinkels eine gut befestige Mauer kaum zerstören. Es wurde mit Leitern, Sturmböcken oder Belagerungstürmen angegriffen, während die Festungsbaumeister die Mauern durch Gräben und Türme, vorgeschobene Befestigungen, gestaffelte Wälle oder innenliegende Zitadellen weiter sicherten. Schon Städte wie Jericho lieferten das Vorbild für viele tausend Jahre Stadtbau. Zwei Meter dicke und sechs Meter hohe Mauern aus Felsblöcken umgaben zusammen mit dreistöckigen Türmen die Stadt. Davor verlief ein drei Meter tiefer und neun Meter breiter Graben. Die Möglichkeit, um im heutigen Jargon zu reden, mit asymmetrischen Mitteln eine Stadt gegen eine überlegene Macht zu verteidigen, konnte so auch das Überleben von Stadtstaaten sichern.

In großen Zentralreichen wie Ägypten, Persien oder Rom waren neue Städte oft nicht durch Mauern gesichert, dafür aber die Grenzen des Reichs mit Festungen und Befestigungsanlagen oder Schutzwällen wie 